Bazer F.W., Thatcher W.W., Martinat-Botté F., Terqui M., 1988a. Conceptus development in Large White and prolific Chinese Meishan pigs. J. Reprod. Fert., 84, 37-44.

Bazer F.W., Thatcher W.W., Martinat-Botté F., Terqui M., 1988b. Sexual maturation and morphological development of the reproductive tract in Large White and prolific Chinese Meishan pigs. J. Reprod. Fert., 83, 723-730.

Billig H., Furuta I., Hsueh A.J.W., 1993. Estrogens inhibit and androgen enhance ovarian granulosa cell apoptosis. Endocrinology, 133, 2204-2212.

Bolet G., Martinat-Botté F., Locatelli A., Gruard J., Terqui M., Berthelot F., 1986. Components of prolificacy in hyperprolific Large White sows compared with the Meishan and Large White breeds. Genet. Sel. Evol., 18, 333-342

Cognié Y., Benoit F., Poulin N., Khatir H., Driancourt M.A., 1998. Oocyte function in sheep ; effect of follicle size and of the FecB Booroola gene. J. Reprod. Fert., 112, 379-386.

Cunningham P.J., England M.E., Young L.D., Zimmerman D.R., 1979. Selection for ovulation rate in swine ; correlated response in litter size and weight. J. Anim. Sci., 48, 509-517.

Driancourt M.A., Terqui M., 1996. Follicular growth and maturation in hyperprolific and Large White sows. J. Anim. Sci., 74, 2231-2238.

Driancourt M.A., Locatelli A., Prunier A., 1995 Effects of gonadotrophin deprivation on follicular growth in gilts. Reprod. Nutr. Dev., 35, 663-673.

Guthrie H.D., Bolt D.J., Cooper B.S., 1990. Effects of gonadotropin treatment on ovarian follicle growth and granulosa cell aromatase activity in prepuberal gilts. J. Anim. Sci., 68, 3719-3726.

Guthrie H.D., Bolt D.J., Cooper B.S., 1993. Changes in follicular ostradiol $17 \beta$, progesterone and inhibin immunoactivity in healthy and atretic follicles during preovulatory maturation in the pig. Dom Anim. Endocr., 10, 127-140.

Hillier S.G., Miro F., 1992. Relative effects of activin and inhibin on steroid hormone synthesis in primate oranulosa cells. J. Clin. Endocr. Metab., 75, 1556 1561.

Howard H.J., Ford J.J., 1992. Relationship among concentrations of steroids, inhibin, IGF1 and IGF binding proteins during follicular development in weaned sows. Biol. Reprod., 47, 193-201.

Kelly C.R., Kopf J.D., Zimmerman D.R., 1988a. Characterization of antral follicle populations during the estrous cycle in pigs selected for ovulation rate. J. Anim. Sci., 66, 1230-1235.
Kelly C.R., Socha T.E., Zimmerman D.R., 1988b. Characterization of gonadotropic and ovarian steroid hormones during the periovulatory period in high ovulating select and control line gilts. J. Anim. Sci. $66,1462-1474$.

Kraeling R.R., Kesner J.S., Estienne M.J., Estienne C.E., Barb C.R., Rampacek G.B., 1990. Follicle growth in hypophysial stalk transected pigs given pulsatile GnRH and PMSG. Dom. Anim. Endocr., 7, 395-402.

Krzymoski T., Kotwica J., Stefanczyk S., Czarnocki J., Debek J., 1982. A sub-ovarian exchange mechanism for the countercurrent transfer of ovarian steroid hormones in the pig. J. Reprod. Fert., 65, 457465 .

Legault C., Gruand J., Bolet G., 1981. Résultats de l'utilisation en race pure et en croisement d'une lignée dite "hyperprolifique ». Journées de la Recherche Porcine en France, 13, 261-267. ITP, Paris.

Montgomery G.W., McNatty K.P., Davis G.H., 1992 Physiology and molecular genetics of mutations that increase ovulation rate in sheep. Endocrine Reviews, $13,309-328$.

Richards J.S., 1980. Maturation of ovarian follicles actions and interactions of pituitary and ovarian hormones on follicular cell differenciation. Phys. Rev., 60, 51-87.

Vanderhyden B.C., Cohen J.N., Morley P., 1993. Mouse oocytes regulate granulosa cell steroidogenesis. Endocrinology, 133, 423-427.

Vatzias G., Knox R., Naber C., Zimmerman D.R., 1991. Further characterization of changes in ovarian follicle populations during the follicular phase as affected by genetic selection for high ovulation rate in gilts. J. Anim. Sci., 69 (suppl. 1), 432 (Abst).

Veldhuis J.D., Klase P.A., Strauss J.F., Hammond J.M., 1982. The role of estradiol as a biological amplifier of the actions of follicle stimulating hormone : in vitro studies in swine granulosa cells. Endocrinology, 111, 144-151.

Yuan W., Lucy M.C., Smith M.F., 1996. Messenger Ribonucleic acid for insulin like growth factors 1 and 2, IGFBP2, gonadotrophin receptors and steroidogenic enzymes in porcine follicles. Biol. Reprod., 55 , 1045-1054.

Zhou J., Adesanya O.O., Vatzias G., Hammond J.M., Bondy C.A., 1996. Selective expression of Insulinlike Growth factor system components during porcine ovary follicular selection. Endocrinology, 137 4893-4901.
M. TERQUI, F. MARTINAT-BOTTÉ

INRA Station de Physiologie de la Reproduction des Mammifères Domestiques, 37380 Nouzilly

\title{
Mortalité embryonnaire et prolificité chez la truie
}

Une ovulation n'est pas obligatoirement associée à la naissance d'un jeune. En fait, celle-ci va dépendre de la survie embryonnaire (nombre d'embryons vivants à 30 jours de gestation par rapport au nombre d'ovulations) et de la survie fotale (nombre de foetus ou de porcelets par rapport au nombre d'embryons). La survie embryonnaire est l'élément principal qui conditionne la taille de la portée et elle varie de façon importante. Ainsi, elle est de 66,68 et $93 \%$ respectivement chez les truies Large White, Large White hyperproli- 
fique et Meishan (Terqui et al 1992 et résultats non publiés). Les facteurs qui affectent cette survie embryonnaire sont nombreux (Hochereau de Reviers et al 1997). On peut les regrouper en trois grandes catégories selon qu'ils agissent sur la qualité des gamètes au moment de la fécondation, le développement des embryons et les interactions entre ces embryons et l'environnement maternel. Parmi ces facteurs, le moment d'ovulation joue un rôle important car il peut affecter à la fois la qualité de l'ovocyte et celle des spermatozoïdes.

\section{1 / Ovulation et qualité de l'ovocyte}

\section{1 / Méthodes de détection de l'ovulation}

La visualisation de l'ovaire est possible par endoscopie (Signoret et al 1972, Brüssow et al 1990) et par échographie (Weitze et al 1989, Sœde et al 1992). Le suivi de la progestérone plasmatique peut être utilisé comme alternative à ces méthodes pour dater a posteriori le moment d'ovulation (Martinat-Botté et al 1995).

L'endoscopie suppose une anesthésie de la femelle. En revanche, l'échographie s'effectue de façon non invasive pour l'animal et permet de visualiser les follicules présents sur les ovaires et ainsi de déterminer le moment d'ovulation (Martinat-Botté et al 1998). Celuici correspond à l'heure de l'examen à laquelle on constate pour la première fois la disparition sur l'écran de l'échographe des taches noires ou follicules. L'échographie, par voie rectale, permet de compter les follicules et d'estimer ainsi la durée de l'ovulation. La détermination du moment d'ovulation par dosage de la concentration de progestérone est basée sur son augmentation dès l'ovula- tion. Dans ce cas, le moment est défini comme étant celui qui correspond à la première valeur supérieure d'un écart type au niveau de base.

Toutes ces techniques supposent de réaliser plusieurs interventions (examens ou prélèvements de sang) pendant toute la durée de l'œstrus pour dater le moment de l'ovulation. La précision du moment d'ovulation déterminée par ces méthodes dépend de la fréquence à laquelle ces examens sont réalisés.

\section{2 / Durée des ovulations}

Il s'agit, pour un même animal, de la durée entre la première et la dernière ovulation. De grandes variations ont été décrites dans la littérature en ce qui concerne la durée de l'ovulation. Celle-ci est en moyenne de 3 à 4 heures (du Mesnil du Buisson et al 1970). L'intervalle entre la première et la dernière ovulation peut durer de moins d'une heure à 7 heures (Flowers et Esbenshade 1993). Ceci influera sur l'état de développement des embryons.

\section{3 / Variabilité du moment d'ovulation}

L'ovulation apparaît en moyenne $43 \pm 13$ heures après le début des chaleurs ; ces résultats ont été obtenus dans 5 élevages (Martinat-Botté et al 1997).

Des différences existent entre races. Ainsi, l'ovulation intervient en moyenne plus tôt après le début de l'œstrus chez les Large White (35 heures) par comparaison aux Meishan (45 heures, Terqui et al 1990). Cette différence de dix heures, en moyenne, dans les moments d'ovulation semble avoir des conséquences sur la qualité des ovocytes de Meishan. Une décharge préovulatoire de LH plus importante (Ellendorff et al 1988) et des niveaux plus élevés d'œstradiol dans les follicules (Hunter et al 1993) ont été décrits chez les Meishan. Ces faits contribuent sans doute

Figure 1. Variabilité de l'intervalle début œstrus-ovulation estimé par la montée de progestérone ( $n=198$, d'après Martinat-Botté et al 1997).

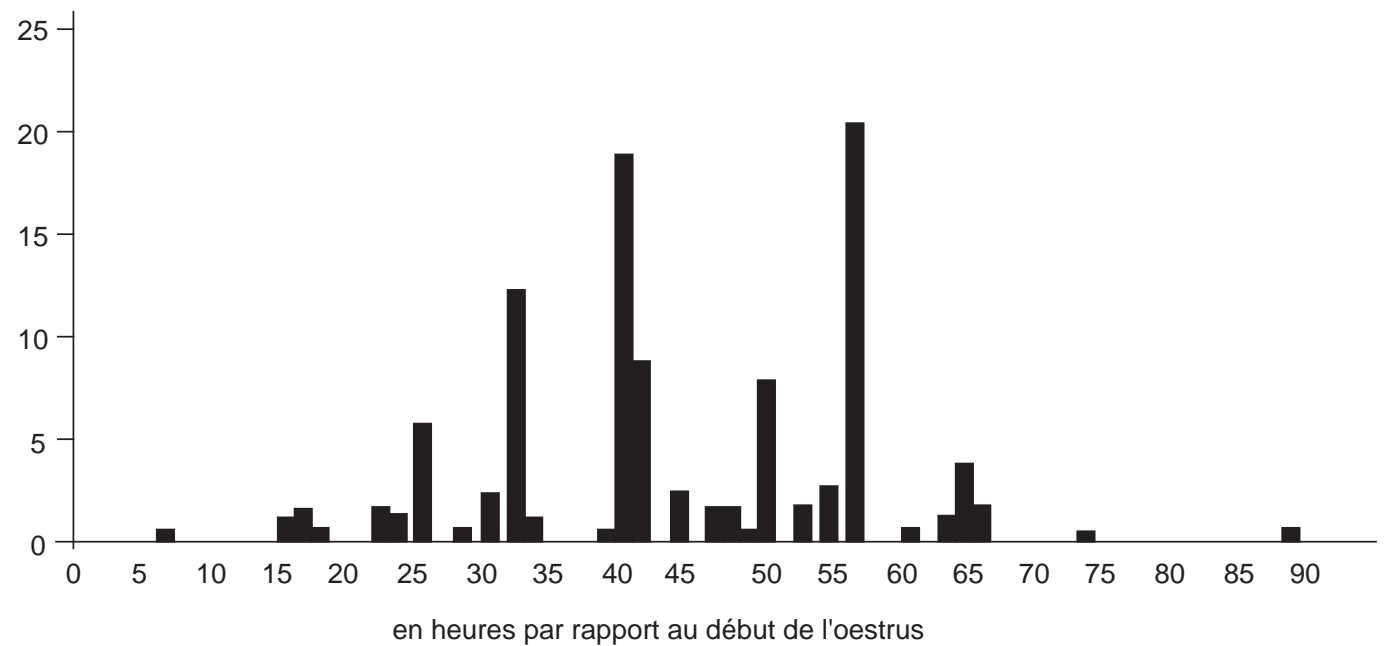


à une meilleure maturation des ovocytes de Meishan et à un développement précoce (stade 2 à 4 cellules) plus rapide de ces embryons (Terqui et al 1992).

Par ailleurs, chez des femelles croisées, l'ovulation commence à des moments très variables par rapport au début de l'œstrus dans l'étude de Martinat-Botté et al (1997), la plus précoce fut observée à 6 heures et la plus tardive à 88 heures (figure 1 ).

Dans les élevages suivis, aucune relation significative n'a été trouvée entre le jour d'apparition de l'œstrus après le tarissement et la période d'ovulation. En effet, une variabilité importante du moment d'ovulation existe pour les différents jours (MartinatBotté et al 1997). Par ailleurs, après un traitement progestatif (Régumate) pour synchroniser les œstrus chez la cochette, la variabilité du moment d'ovulation par rap-

Tableau 1. Diamètre (moyenne \pm écart type) de l'embryon Meishan (MS) et Large White (LW) entre 8 et 11 jours de gestation (d'après Bazer et al 1988). $n$ : nombre de cochettes gravides.

\begin{tabular}{|c|c|c|c|}
\hline Stade de gestation & Paramètres & MS & LW \\
\hline 8 jours & $\begin{array}{l}\text { Diamètre moyen de l'embryon (mm) } \\
\text { Coefficient de variation }(\%)\end{array}$ & $\begin{array}{c}0,45 \pm 0,1 \\
(\mathrm{n}=4) \\
20\end{array}$ & $\begin{array}{c}0,69 \pm 0,3 \\
(\mathrm{n}=4) \\
46\end{array}$ \\
\hline 10 jours & $\begin{array}{l}\text { Diamètre moyen de l'embryon (mm) } \\
\text { Coefficient de variation }(\%)\end{array}$ & $\begin{array}{c}2,7 \pm 0,8 \\
(\mathrm{n}=4) \\
29\end{array}$ & $\begin{array}{c}1,9 \pm 0,7 \\
(\mathrm{n}=4) \\
38\end{array}$ \\
\hline 11 jours & $\begin{array}{l}\text { Diamètre moyen de l'embryon (mm) } \\
\text { Coefficient de variation }(\%)\end{array}$ & $\begin{array}{c}5,3 \pm 1,2 \\
(\mathrm{n}=5) \\
22\end{array}$ & $\begin{array}{c}2,7 \pm 1,2 \\
(\mathrm{n}=4) \\
\quad 42\end{array}$ \\
\hline
\end{tabular}

Figure 2. Relation entre l'intervalle insémination-ovulation et le nombre total de porcelets nés (d'après Nissen et al 1997, (c) Elsevier Science Inc. 1997).

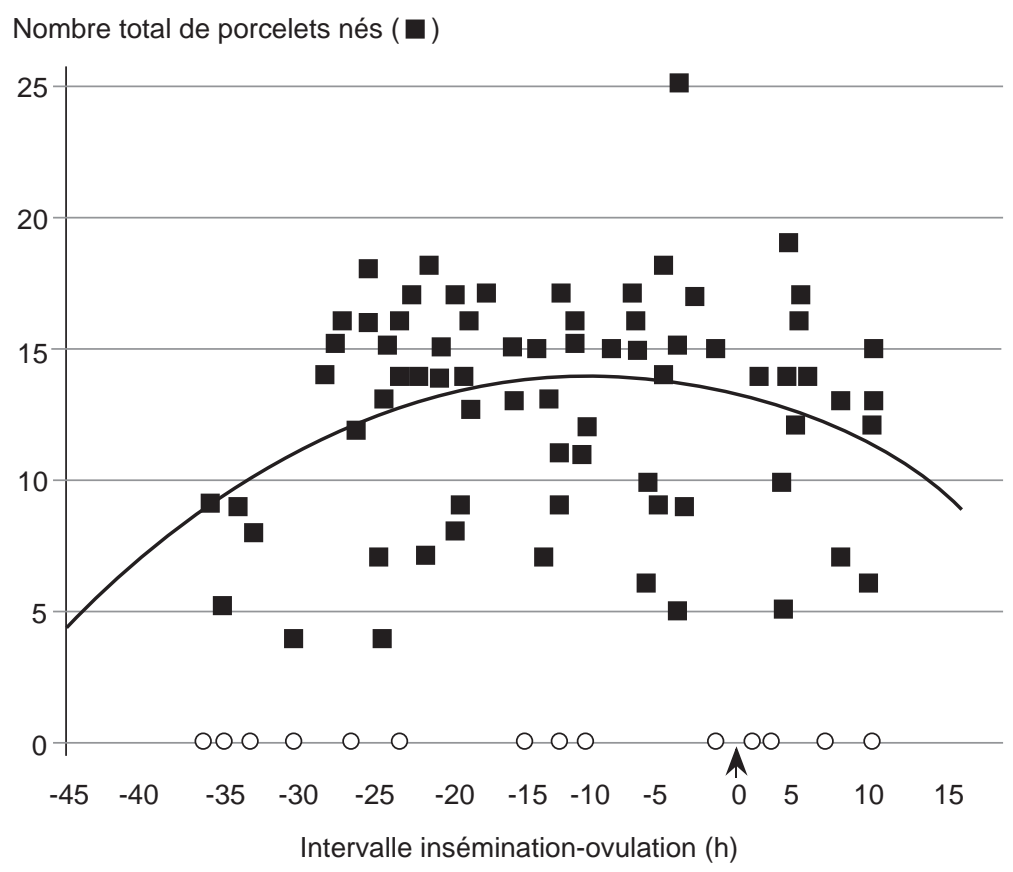

O Truies vides port au début de l'œstrus est similaire à celle de femelles non traitées (Martinat-Botté et al 1995 et 1997).

\section{4 / Conséquences du moment d'ovulation et d'insémination sur la fertilité et la taille de la portée}

Lorsqu'une insémination en semence fraîche ou congelée est réalisée entre - 12 heures et +4 heures par rapport à l'ovulation, le taux de fécondation est supérieur à $90 \%$ (Waberski et al 1994). Des résultats similaires ont été obtenus par Sœde et al (1995).

Pour apprécier les conséquences de la variabilité du moment d'ovulation, l'intervalle IA-ovulation est retenu comme critère. Or, en élevage, les truies sont inséminées plusieurs fois au cours de l'œstrus, on est donc amené à retenir l'intervalle minimum entre l'ovulation et l'insémination. Un effet significatif sur la taille de la portée n'est pas toujours observé (Martinat-Botté et al 1997, Nissen et al 1997), mais, dans ces deux études, un accroissement est noté lorsque l'insémination est réalisée près du moment d'ovulation (figure 2). Le numéro d'insémination "supposée fécondante " affecte la taille de la portée. Une augmentation significative de deux porcelets en moyenne est observée selon que la première ou la seconde insémination est la plus proche de l'ovulation (Martinat-Botté et al 1997).

\section{2 / Le développement embryonnaire}

Une série d'expériences a permis de comparer le développement embryonnaire à partir de la fécondation jusqu'à l'implantation chez les animaux Large White et Meishan.

Pour Pope et al (1990), l'homogénéité de taille des embryons serait déterminante pour la survie embryonnaire. Les embryons les plus avancés empêchent la survie de ceux qui sont en retard. Chez le génotype Meishan, la survie embryonnaire est supérieure à celle des races européennes (Terqui et al 1990, Haley et Lee 1993) et le développement embryonnaire apparaît plus homogène (tableau 1, Bazer et al 1988). Toutefois, Youngs et al (1993) n'ont pas retrouvé ces observations. Cependant, le schéma d'insémination utilisé par ces auteurs conduit chez la Meishan à un écart IA-ovulation trop grand, ce qui a vraisemblablement réduit le pourcentage d'embryons normaux (Sœde et al 1995). L'homogénéité chez la Meishan (Bazer et al 1988) pourrait être le résultat d'un intervalle entre la première et la dernière ovulation plus court que chez la truie Large White (Martinat-Botté et al 1987) 


\section{3 / Les interactions entre l'embryon et l'environnement maternel}

\section{1 / La nature des interactions}

Le signal embryonnaire le mieux connu est l'œstradiol $17 \beta$ qui est produit dès le $10^{\mathrm{e}}$ jour. Il empêche la régression du corps jaune et induit le maintien de la gestation. De très nombreux facteurs de croissance et de différenciation et leurs récepteurs ont été mis en évidence dans l'embryon, l'oviducte, l'utérus. Ainsi, par exemple, le complexe IGF-I, IGF-II, récepteurs à IGF-I, IGF-II, et à l'insuline a-til été mis en évidence dans l'oviducte, l'utérus et le blastocyste (Simmen et al 1992, Chastant et al 1994). Ce complexe interviendrait dans la régulation de la production d'œstradiol $17 \beta$. Le LIF (Leukemia Inhibitory Factor) est produit par l'utérus au cours de la période péri-implantatoire (Anegon et al 1994). Ce composé joue un rôle chez la souris dans le mécanisme d'implantation (Stewart et al 1992). Dans un milieu de culture qui contient du LIF, les cellules embryonnaires souches porcines restent indifférenciées (Hochereau de Reviers et al 1993) ; de plus, les cellules du bouton embryonnaire possèdent des récepteurs aux LIF (Wianny et al 1995). Des différences de teneurs ou d'expression ont été observées entre Large White et Meishan pour un certain nombre de ces facteurs (Simmen et al 1992). Cependant, le rôle de ces facteurs dans la survie embryonnaire n'est pour l'instant pas établi chez le Porc.

\section{2 / Les paramètres morphologiques du tractus génital}

La taille du tractus génital est déterminante pour la croissance des fœetus. Jusqu'à 30 jours de gestation, le poids et la longueur de l'utérus rapporté au poids du corps ne sont pas différents entre les femelles Large White et les femelles Meishan (Bazer et al 1988). En revanche, chez la truie Large White hyperprolifique, les cornes utérines sont nettement plus longues que celles des deux autres génotypes (M. Terqui, résultats non publiés). Cet accroissement pourrait résulter de taux plus élevés d'œstrogènes et de progestérone consécutifs au plus grand nombre d'ovulations.

\section{3 / Le transfert d'embryons}

Le transfert d'embryons d'une truie d'un génotype dans une receveuse d'un autre génotype est un moyen d'évaluer les interactions entre l'embryon et l'environnement maternel. Les résultats obtenus par les différentes équipes sont assez contradictoires. Ashworth et al (1990) observent un effet défavorable de l'environnement maternel Meishan. Youngs et al (1994) n'observent pas de différence, mais les embryons transférés avaient été récupérés au tout début de leur implantation. Le taux
Tableau 2. Réussite du transfert d'embryons ( $n=$ nombre de transferts) chez deux génotypes (d'après Martinat Botté et al 1993).

\begin{tabular}{|lcc|}
\hline Génotype de l'embryon & MS X LW & Croisé \\
Receveuse & MS & Croisée \\
Taux de gestation à $30 \mathrm{j}$ & $83 \%$ & $54,2 \%$ \\
& $(\mathrm{n}=48)$ & $(\mathrm{n}=48)$ \\
\hline
\end{tabular}

de réussite de transfert d'embryons est meilleur lorsque des embryons croisés Meishan sont transférés dans des receveuses Meishan comparé à celui obtenu après transfert d'embryons de lignée européenne dans des truies de même génotype (tableau 2, Martinat-Botté et al 1993).

\section{Conclusion}

La mortalité embryonnaire peut avoir des causes multiples, les unes liées à l'ovocyte et à l'ovulation, les autres dépendant du développement de l'embryon et aussi des interactions entre le compartiment maternel et les embryons. L'importance quantitative de ces interactions dans le déterminisme de la survie embryonnaire reste à établir. L'analyse des mécanismes sous-jacents (maturation ovocytaire, facteurs de croissance...) devrait permettre de mieux comprendre comment ces interactions s'organisent dans l'espace et dans le temps. Toutefois, il semble possible à court terme de réduire la variabilité du moment d'ovulation et ainsi de limiter la mortalité embryonnaire et augmenter la prolificité.

\section{Références}

Ashworth C.J., Haley C.S., Aitken R.P., Wilmut I., 1990. Embryo survival and conceptus growth after reciprocal embryo transfer between Chinese Meishan and Landrace x Large White gilts. J. Reprod. Fert., 90, 595-603.

Anegon I., Cuturi M.C., Godard A., Moreau M., Terqui M., Martinat-Botté F., Soulillou J.P., 1994. Presence of leukemia inhibitory factor and interleukin 6 in porcine uterine secretions prior to conceptus attachment. Cytokine, 6, 493-499.

Bazer F.W., Thatcher W.W., Martinat-Botté F., Terqui M., 1988. Conceptus development in Large White and prolific Chinese Meishan pigs. J. Reprod. Fert., $84,37-42$

Brüssow K.P., Ratky J., Becker F., 1990. Determination of the duration of ovulation in gilts by means of laparoscopy. Reprod. Dom. Anim., 25, 184-190.

Chastant S., Monget P., Terqui M., 1994. Localization and quantification of insulin-like growth factor-I (IGF-I) and IGF-II/ mannose-6-phosphate (IGFII/M6P) receptors in pig embryos during early pregnancy. Biol. Reprod., 51, 588-596. 
Ellendorff F., Martinat-Botté F., Signoret J.P., Terqui M., 1988. Duration of œstrus and preovulatory LH in the high fertility Meishan pig. 11th Int. Cong. Anim. Reprod. Artif. Insem., 26-30 June 1988, Dublin, Ireland. Vol 2, 24 (Abstract).

Flowers W.L., Esbenshade K.L., 1993. Optimizing management of natural and artificial matings in swine. J. Reprod. Fert., Suppl. 48, 217-228.

Haley C.S., Lee G.J., 1993. Genetics basis of prolificacy in Meishan pigs. J. Reprod. Fert., Suppl. 48, 247-259.

Hochereau de Reviers M.T., Perreau C., 1993. In vitro culture of embryonic disc cells from porcine blastocysts. Reprod. Nutr. Dev., 33, 475-483.

Hochereau de Reviers M.T., Lagant H., Desprès P., Venturi E., Brunet L., Perreau C., Wianny F., Legault C., 1997. Facteurs de variation de l'âge à la puberté, du taux d'ovulation et de la survie embryonnaire précoce chez la cochette : type génétique, poids vif, saison et consanguinité. Journées Rech. Porcine en France, 29, 109-113.

Hunter M.G., Biggs C., Faillace L.S., 1993. Endocrine and follicular studies in Meishan pigs. J. Reprod. Fert., Suppl. 48, 261-270.

Martinat-Botté F., Bazer F.W., Thatcher W.W., Terqu M., Locatelli A., Chupin D., 1987. Oestrus, ovulation, conceptus and uterine development in Large White (LW) and hyperprolific Chinese Meishan (MS) gilts. Annual Conference of the Society for the Study of Fertility, July 1987, York (G.B.), 44 (Abstract).

Martinat-Botté F., Procureur R., Plat M., Forgerit Y., Bussière J., Bariteau F., Desprès P., Locatelli A., Terqui M., 1993. Embryo transfer in the pig : Effects of the number of embryos transferred and genotype. Colloque de l'Association Européenne de Transfert Embryonnaire (AETE), 10-11 septembre 1993, Lyon (France), 236 (Abstract).

Martinat-Botté F., Richard D., Maurel M.C., Plat M., Desprès P., Locatelli A., Godet G., Landrevi J., Bussière J., Renaud G., Terqui M., 1995. Relations entre les taux plasmatiques de LH, de progestérone, l'échographie d'ultrasons et le moment d'ovulation chez la cochette. Journées Rech. Porcine en France, 27, $57-62$.

Martinat-Botté F., Forgerit Y., Maurel M.C., Corbe H., Bernelas D., Mercat M.J., Guillouet P., Terqu M., 1997. Etude des moments d'ovulation et d'insémination chez la truie et de leurs conséquences sur la taille de la portée en élevages. Journées Rech. Porcine en France, 29, 103-108.

Martinat-Botté F., Renaud G., Madec F., Costiou P., Terqui M., 1998. Echographie et reproduction chez la truie. INRA Editions et Hoechst Roussel Vet., Paris, $103 \mathrm{p}$.

du Mesnil du Buisson F., Mauléon P., Locatelli A., Mariana J.C., 1970. Modification du moment et de l'étalement des ovulations après maîtrise du cycle sexuel de la truie. In : R. Palmer (ed), Inhibition de l'ovulation, 225-234. Masson, Paris.

Nissen A.K., Sœde N.M., Hyttel P., Schmidt M., D'hoore L., 1997. The influence of time of insemination relative to time of ovulation on farrowing frequency and litter size in sows as investigated by ultrasonography. Theriogenology, 47, 1571-1582.
Pope W.F., Xie S., Brœrmann D.M., Nephew K.P., 1990. Causes and consequences of early embryonic diversity in pigs. J. Reprod. Fert., Suppl. 40, 251-260.

Signoret J.P., du Mesnil du Buisson F., Mauléon P., 1972. Effect of mating on the onset and duration of ovulation in the sow. J. Reprod. Fert., 31, 327-330.

Simmen F.A., Simmen R.C.M., Geisert R.D., Martinat-Botté F., Bazer F.W., Terqui M., 1992. Differential expression, during the estrus cycle and pre- and postimplantation conceptus development, of messenger ribonucleic acids encoding components of the pig uterine insulin-like growth factor system. Endocrinology, 130, 1547-1556.

Sœde N.M., Noordhuizen J.P.T.M., Kemp B., 1992. The duration of ovulation in pigs, studied by transrectal ultrasonography, is not related to early embryonic diversity. Theriogenology, 38, 653-666.

Sœde N.M., Wetzels C.C.H., Zondag W., Hazeleger W., Kemp B., 1995. Effects of a second insemination after ovulation on fertilization rate and accessory sperm count in sows. J. Reprod. Fert., 105, 135-140.

Stewart C.L., Kaspar P., Brunet L.J., Bhatt H., Gadi I., Kontgen F., Abbondanzo S.J., 1992. Blastocyst implantation depends on maternal expression of leukaemia inhibitory factor. Nature, 359, 76-79.

Terqui M., Bazer F.W., Martinat-Botté F., 1990 Quels sont les mécanismes responsables de la prolificité de la race Meishan? In : Symposium sur le Porc Chinois, 5-6 Juillet 1990, Toulouse (France), 19-32. INRA, Paris.

Terqui M., Bazer F.W., Martinat-Botté F., 1992 Mechanisms of high embryo survival in Meishan gilts. In : International Symposium on Chinese Pig breeds, 11-14 août 1992, Harbin, China, 52-58.

Waberski D., Weitze K.F., Gleumes T., Schwartz M. Willmen T., Petzholdt R., 1994. Effects of time of insemination relative to ovulation on fertility with liquid and frozen boar semen. Theriogenology, 42 831-840.

Weitze K.F., Habeck O., Willmen T., Rath D., 1989 Detection of ovulation in the sow using Transcutaneous Sonography. Zuchthyg., 24, 40-42.

Weitze K.F., Wagner-Rietschel H., Waberski D., Richter L., Krieter J., 1994. The onset of heat after weaning, heat duration and ovulation as major factors in AI timing in sows. Reprod. Dom. Anim., 29, 433-443.

Wianny F., Van Eijk M., Mummery C.L., Hochereau de Reviers M.T., 1995. Characterisation of the cells derived from porcine embryonic discs in culture-Role of LIF. Society for Study of Fertility (SSF), Montpellier, France.

Youngs C.R., Ford S.P., McGinnis L.K., Anderson L.H., 1993. Investigations into the control of litter size in swine. I. Comparative studies on in vitro development of Meishan and Yorkshire preimplantation embryos. J. Anim. Sci., 71, 1561-565.

Youngs C.R., Christenson L.K., Ford S.P., 1994 Investigations into the control of litter size in swine. III. A reciprocal embryo transfer study of early conceptus development. J. Anim. Sci., 72, 725-731. 